\title{
Index to genera and species, Volume 1 (1990)
}

\section{Acarina}

Ameronothus dubidini 197

Cymbaeremaeus cymba 197

Gobiella Balogh \& Mahunka 103

Graptoppia foveolata (Balogh) 104, 105

- tanaitica Karppinen \& Poltavskaja sp. n. 104, 105

Infernobates Karppinen \& Poltavskaja gen. n. 103

- citelli Karppinen \& Poltavskaja sp. n. 103, 104

Kaszabobates Balogh 103

- kaszabi (Balogh \& Mahunka) 103

Medioppia Subias \& Minguez 129

- centrodentata Gordeeva \& Niemi sp. n. 129, 130

- media (Michelcic) 129

- minidentata Subias 129

- tridentata Subias 129

Oppia fallax var. obsoleta Paoli 130

Oppia media Michelcic 129

Zetorchestes phylliterus Mahunka 106

- reticulatus Karppinen \& Poltavskaja sp. n. 105, 106

\section{Coleoptera}

Abropus carnifex (Fabricius) 3, 4, 8-12, 15

Acrotona pusilla (Brundin) 110

Aemalodera centromaculata Solier 9, 12, 15

- testacea (Jeannel) 9, 12, 15

Agelastica Chevrolat 120

Ahasverus advena (Waltl) 110

Amara brunnea (Gyllenhal) 235

- littorea Thomson 110

Antarctonomus complanatus (Blanchard) 3, 9-11, 15

Aplosonyx chalybeus (Hope) 203

Asemum striatum (Linnaeus) 171-173

Astenus pulchellus (Heer) 110

Atheta coriaria (Kraatz) 110

- xanthopus (Thomson) 110

Atholus bimaculatus (Linnaeus) 110

- duodecimstriatus (Schrank) 110

Atysa marginata (Hope) 202

Barypus clivinoides (Curtis) 9, 10, 15

Bembidiomorphum convexum Champion 9, 10, 15

Bohemiellina flavipennis (Cameron) 110

Buprestis haemohorrdalis Herbst 221

- tarda Fabricius 222

Caenoscelis subdeplanata Brisout de Barneville 110

Calathus micropterus (Duftschnid) 234-236

Carabus hortensis Linnaeus 235

Carpophilus hemipterus (Linnaeus) 110

- marginellus Motschulsky 110

Cascellius aeneoniger Waterhouse 9-11, 15

- gravesii Curtis 3, 8, 9-11, 15

- hyadesi Fairmaire 9, 10, 15

Catops fuscus (Panzer) 110

Cephaloon Newman 120

Cercyon laminatus (Sharp) 110

Ceroglossus suturalis (Fabricius) 3, 4, 9-12, 15
Chrysomela vigintipunctata (Scopoli) 208

Cryptophagus laticollis Lucas 110

Cychrus caraboides (Linnaeus) 235, 236

Dendroctonus valens LeConte 171-173

Dercetina histrio Baly 203

Dinothenarus pubescens (De Geer) 110

Diorhabda lusca Maulik 202

Doryxena grossa (Hope) 202

Erirhinus Schönherr 120

Galerucella distincta (Baly) 202

- grisescens (Joann.) 202

- placida Baly 202

Galleruca trifasciata Fabricius 206

- trifasciata Hope 206

Gallerucida indica Harold 204

Gauropterus fulgidus (Fabricius) 110

Geotrupes stercorarius (Linnaeus) 107-110

- stercorosus (Scriba) 107, 108, 110

- vernalis (Linnaeus) 107, 108, 110

Hister funestus Erichson 110

Hylaspes Baly 120

- dohrni Duvivier 204

- longicornis Baly 204

Hylobius pales (Herbst) 171-174

Kenodactylus audouini (Guérin) 12

Leistus terminatus (Hellwig) 235-237

Leptacinus batychrus (Gyllenhal) 110

Leptarthra fasciata Jacoby 206

Leptodirus Schmidt 119

Lithocaris ochracea (Gravenhorst) 110

Margarinotus ventralis (Marseul) 110

Melanophila acuminata De Geer 222

- cyanea (Fabricius) 221-225

- cyanea aerea (Ganglbauer) 222

- cyanea cyanea (Fabricius) 221

- formaneki (Jakobson) 221-225

- formaneki bohemica (Bíly) 222

- formaneki formaneki (Jakobson) 222

- formaneki lavagnei (Thery) 222

Menippus cervicus (Hope) 202

Meristata dohrni (Baly) 205

- fallax (Harold) 205

- pulunini (Bryant) 205

- quadrifasciata (Hope) 205

- quadrifasciata interrupta (Kollar \& Redtenbacher) 205

- sexmaculata (Kollar \& Redtenbacher) 206

- spilota (Hope) 206

Metius sp. 9,15

- blandus (Dejean) 9, 10,15

- bradytoides (Fairmaire) 9,15

Migadops latus (Guérin-Méneville) 3, 8, 9-12, 15

Monachulus Leng 120

Monachus Chevrolat 120

Monochamus spp. 173

- scutellatus (Say) 171-174

Monotoma bicolor Villa 110

Nepalogaleruca elegans Kimoto 206 
Nepalogaleruca elegans angustiiineata Kimoto \& Takizawa 206

Notaris Germar 120

Nothotrechisibus hornensis (Fairmaire) 9, 15

Notiophilus biguttatus (Fabricius) 235, 236

Oides flava (Oliver) 201

- livida (Fabricius) 201

- scutellata (Hope) 201

Oopterus clivinoides Guérin 12

- soledadius (Guérin) 9-12, 15

Oxypteris acuminata (DeGeer) 222

Oxytelus migrator Fauvel 110

Pallasiola theoneiformis Mandl 203

Patrobus atrorufus (Ström) 235

Periclitena vigorsii (Hope) 202

Perigona nigriceps (Dejean) 110

Philothus discoideus (Gravenhorst) 110

- longicornis Stephens 110

- parvicornis (Gravenhorst) 110

- pseudovarians Strand 110

Pissodes piniphilus (Herbst) 224

Plagiotelum irinum Solier 9, 15

Pseudadimonia variolosa (Hope) 203

Pseudoides flavovittis (Motschulsky) 203

Pseudomedon obscurella (Erichson) 110

Pterostichus melanarius (Illiger) 235

- oblongopunctatus (Fabricius) 234-237

Ptilium minutissimum (Ljungh) 110

Pycnochila fallaciosa Chevrolat 9, 12, 15

Pytho sp. 171-173

Rhagium inquisitor (Linnaeus) 171-174

- inquisitor (Linnaeus) 224

Rugilus scutellatus (Motschulsky) 110

Sastroides purpurascens (Hope) 202

Scymnus capicola Casey 163, 165, 168, 169

- congoanus Mader 163, 166

- cyclicus Fürsch 163, 168, 169

- danielssoni Fürsch sp. n. 163-165, 168, 169

- declaratus Mader 163-165, 168, 169

- demeteri Fürsch 163, 164, 167-170

- exasparans Pope 164

- kibaliensis Fürsch sp. n. 163-166, 168, 169

- kibonotensis Weise 163, 166-170

- nummelini Fürsch sp. n. 163, 167-169

- patruelis Mader 163, 168, 169

- problematicus Mader 164

- scapuliferus Mulsant 163, 167, 169

- scapuliferus posticus Wollaston 169

- scapuliferus scapuliferus Mulsant 168, 169

- semidivisus Mader 166

- simulans 163, 167, 169

Sermyla Chapuis 120

Sermylassa Reitter 120

Sphenoraia bicolor (Hope) 203

- rutilans (Hope) 204

Stagobium Schiödte 119

Stenotrachelus Latreille 120

Tachinus lignorum (Linnaeus) 110

- marginatus Gyllenhal 110

Temnostega antarctica Enderlein 12

Tetropium gabrieli Weise 118

Thecturota marchii (Dodero) 110

Thes bergrothi (Reitter) 110

Tomicus piniperda (Linnaeus) 224, 225

Trechisibus sp. 9, 15

- antarcticus Dejean 8

- australis Jeannel 8

- germaini Jeannel 8

- pernitidus Jeannel 8

Trechus secalis 235
Trirammatus sp. 9, 15

Xanthogaleruca luteola (Müller) 202

- luteola bicarinata (Lopatin) 202

\section{Diptera}

Aenictomyia Brues 33

Aenigmatias lubbocki (Verrall) 28

Aenigmatistes foveolatus Schmitz 28

Aphanotrigonum favillaceum Becker 227, 230, 231

Assuania thalhammeri Strobl 228, 231

Atherix ibis (Fabricius) 113-116

- variegata (Walker 114, 116

Camarota curvipennis Latreille 227, 231

Cataclinusa pachycondylae (Brues) 28

Chrysomya marginalis Wiedemann 151

- megacephala (Fabricius) 151-153

- regalis Robineau-Desvoidy 151-153

Diplonevra Lioy 33, 34, 128

- abbreviata (von Roser) 28

- assmuthi (Schmitz) 35

- bifasciata (Walker) 28, 35

- bilineata (Borgmeier) 33-35

- caudata (Schmitz) 35

- compressicauda Beyer 35

- concinna (Meigen) 28

- evanescens (Brues) 35

- fasciiventris (Brues) 35

- florea (Fabricius) 28

- funebris (Meigen) 28

- glabra Schmitz 28

- gynaptera Fuller \& Lee 33-35

- hyalizona Beyer 35

- ismayi Disney sp. n. 33, 35, 38

- lucida Beyer 35

- mortimeri Disney 33-35, 38

- nigrita (Malloch) 35

- nigroscutellata (Malloch) 35

- novaguineae Beyer 35

- peregrina (Wiedemann) 35, 36

- predicta Disney sp. n. 33, 35, 37, 38

- rufivenia Borgmeier 35

- varians Beyer 35

- watsoni Disney 28, 34, 35, 38

Dohrniphora Dahl 33, 34, 38

Dohrniphoroidea Assmuth 34

- cornuta (Bigot) 28

Elachiptera bimaculata Loew 227, 228

- megaspis Loew 227, 229

Endonepenthia Schmitz 26

Epicnemis flavidula (Brues) 28

Epimegaselia Beyer 25, 29, 30

Fiebrigella sp. 230

Hemiplastophora Beyer 30

Ibisia marginata (Fabricius) 113, 115, 116

Megaselia Rondani 128

- bisetigera (Beyer) 29

- bruesi Disney 26, 28

- caudalis (Beyer) 29

- congrex (Beyer) 29

- furvicolor (Beyer) 29, 30

- rotundicauda (Beyer) 29, 30

- subulicauda Schmitz 30

- torautensis Disney sp. n. 25-29

Meromyza sp. 231

Metaplastophora Beyer 25, 29, 30

Metopina ciceri Disney 33

Multinevra macropygidia Disney 28

Myopiomyia Disney 33 
Myopiomyia harmani Disney 28

Myospila bimaculata (Macquart) 154

- meditabunda (Fabricius) 154

Ortalischema albitarse (Zetterstedt) 154

Oscinella frit Linnaeus 228, 231

Oscinimorpha arcuata Duda 227, 230

Palpiclavina tonkinensis Silvestri 28

Plastophora Brues 26

Plectanocnema nudipes (Becker) 28

Plesioclythia argyrogyna (De Meijere) 28

Polyodaspis sulcicollis Meigen 228, 230

Puliciphora rosei Disney 22

Ouasipseudacteon Beyer 25, 29, 30

Rhynchomicropteron Annandale 21, 128

- aphidiforme Schmitz \& Mjöberg 21-23

- beaveri (Disney) 21

- brevipes Papp 21

- caecutiens Schmitz 21

- caecutiens Silvestri 23

- dudichi Papp 21

- muluensis Disney 21

- necaphidiforme Disney sp. n. 21-23

- nudiventer Papp 21

- puliciforme Annandale 21

- silvestrii Disney sp. n. 21, 23

- vallacki Disney 21

Sciadocera rufomaculata White 28

Scoliophthalmus trapezoides Becker 227

Sepsis biflexuosa Strobl 154

- luteipes Melander \& Spuler 154

- nigripes Meigen 154

- thoracica (Robineau-Desvoidy) 154

Siphunculina ornatifions Loew 227, 229

Speccafrons cypria Nartshuk sp. n. 227, 229

- halophila Duda 229, 230

Tarsophoromyia Beyer 25, 29

Thaumatomyia notata Meigen 227, 228, 231

- sulcifrons Becker 228, 232

Themira biloba Andersson 154

- germanica Duda 154

- lucida (Staeger) 154

- malformans Melander \& Spuler 154

- paludosa Elberg 154

Trachysiphonella pori Harkness \& Ismay 227, 230

Tricimba humeralis Loew 228, 230

- meridiana Dely-Draskovits 227, 231

Woodiphora Schmitz 28

- biroi (Brues) 28

- papuana Disney 28

- parvula Schmitz 28

- retroversa (Wood) 28

- salomonis Beyer 28

\section{Heteroptera}

Acalypta carinata (Panzer) 214

- gracilis (Fieber) 214

- marginata (Wolff) 214

- nigrina (Fallén) 214

- parvula (Fallén) 214

- platycheila (Fieber) 214

Acanthosoma haemorrhoidale (Linnaeus) 214

Acompocoris alpinus Reuter 214

- pygmaeus (Fallén) 214

Acompus rufipes (Wolff) 214

Actinocoris signatus Reuter 214

Adelphocoris lineolatus (Goeze) 214

- quadripunctatus (Fabricius) 214

- seticornis (Fabricius) 214
Adomerus biguttatus (Linnaeus) 214

Aelia acuminata (Linnaeus) 214

- klugi Hahn 214

Aeschyntelus maculatus (Fieber) 214

Agnocoris rubicundus (Fallén) 214

Agramma femorale Thomson 214

- tropidopterum Flor 214

Alloeotomus germanicus E. Wagner 214

- gothicus (Fallén) 214

Alydus calcaratus (Linnaeus) 214

Aneurus avenius (Dufour) 214

Anthocoris confusus Reuter 214

- gallarumulmi (De Geer) 214

- limbatus Fieber 214

- nemoralis (Fabricius) 214

- nemorum (Linnaeus) 214

- sibiricus Reuter 214

- simulans Reuter 214

Aphanus rolandri (Linnaeus) 214

Aphelocheirus aestivalis (Fabricius) 214

Aradus angularis J. Sahlberg 214

- anisotomus Puton 214

- aterrimus Fieber 214

- betulae (Linnaeus) 214

- betulinus Fallén 214

- bimaculatus Reuter 214

- brevicollis Fallén 214

- cinnamomeus (Panzer) 214

- corticalis (Linnaeus) 214

- crenaticollis R.F. Sahlberg 214

- depressus (Fabricius) 214

- erosus Fallén 214

- laeviusculus Reuter 214

- lugubris Fallén 214

- pictus Bärensprung 214

- signaticornis R.F. Sahlberg 214

- truncatus Fieber 214

Arctocorisa carinata (C. Sahlberg) 214

- germari (Fieber) 214

Atomophora Reuter 45, 46, 53

- arabica Linnavuori 47, 49, 51

- basipunctata Wagner 47, 50, 52

- eximia Reuter 46,47

- macrophthalma Poppius 45, 47, 49, 51

- maculosa erato Linnavuori 47, 50

- maculosa maculosa Reuter 50

- maculosa Reuter 45-48

- nut Linnavuori 47, 49, 50, 51

- oculata Reuter 45, 46, 48, 50, 51

- pantherina Reuter 45-47, 49, 50-52

- pentheeus Linnavuori 47-51

- subpallida Wagner 47, 50, 53

Atomoscelis antennalis Lindberg 127

Atractotomus magnicornis (Fallén) 214

- mali (Meyer-Dür) 214

- morio J. Sahlberg 210, 214

Berytinus clavipes (Fabricius) 214

- crassipes (Herrich-Schäffer) 214

- minor (Herrich-Schäffer' 214

- signoreti (Fieber) 214

Blepharidopterus angulatus (Fallén) 214

Bothynotus pilosus (Boheman) 214

Brachyarthrum limitatum Fieber 214

Brachycarenus tigrinus (Schilling) 214

Bryocoris pteridis (Fallén) 214

Callicorixa praeusta (Fieber) 214

- producta (Reuter) 214

- wollastoni (Douglas \& Scott) 214

Callisphodrus Stål 139

Calocoris biclavatus (Herrich-Schäffer) 214 
Calocoris fulvomaculatus (De Geer) 214

- norvegicus (Gmelin) 215

- ochromelas (Gmelin) 215

- roseomaculatus (De Geer) 215

- sexguttatus (Fabricius) 215

Camptotylidea Wagner 45, 46, 53, 63

- alba (Reuter) 45, 54, 55

- albovittata (Reuter) 45, 54, 55

- alhagii (Linnavuori) 45, 51. 54, 56, 58, 60

- astarte (Linnavuori) 45, 53, 54, 57, 60, 61, 62, 63

- astragalii (Linnavuori) 45, 54, 55, 60

- bast (Linnavuori) 45, 54, 57

- bipunctata (Reuter) 45, 54-56

- candida (Linnavuori) 45, 51, 54, 57, 59

- flavescens (Puchkov) 45, 54, 59,60

- fuscomaculata (Reuter) 45, 54, 56, 57, 62

- lineata (Reuter) 45, 53, 56, 58

- modesta (Linnavuori) 45, 54, 60, 61

- pallescens (Puchkov) 45, 54, 60

- persica Wagner 53, 54, 56, 57

- suturalis (Reuter) 45, 51, 54, 56, 58, 60, 62

- vitticollis (Reuter) 45, 53, 54, 56, 57, 62

Camptozygum aequale (Villers) 215

Campylomma verbasci (Meyer-Dür) 215

Capsus ater (Linnaeus) 215

- wagneri Remane 215

Carpocoris purpureipennis (De Geer) 215

Catoplatus fabricii (Stål) 215

Ceratocombus brevipennis Poppius 215

- coleoptratus (Zetterstedt) 215

- corticalis Reuter 215

Charagochilus gyllenhali (Fallén) 215

Chartoscirta cincta (Herrich-Schäffer) 215

- elegantula (Fallén) 215

Chilacis typhae (Perris) 215

Chiloxanthus stellatus (Curtis) 215

Chlamydatus opacus (Zetterstedt) 215

- pulicarius (Fallén) 215

- pullus (Reuter) 215

- saltitans (Fallén) 215

- wilkinsoni (Douglas \& Scott) 210, 215

Chlorochroa juniperina (Linnaeus) 215

- pinicola (Mulsant) 215

Chorosoma schillingii (Schummel) 215

Cimex columbarius Jenyns 215

- lectularius Linnaeus 215

Compsidolon Reuter 123

- maculiiorne Linnavuori 123

- salicellus (Herrich-Schäffer) 215

- hymelaeae Wagner 124

Compsonannus Reuter 123, 128

- hesione Linnavuori sp. n. 123-127

- longicornis Wagner 124-127

- maculicornis Linnavuori 123, 124, 126, 127

- ovatus Wagner 124, 126, 127

- puncticornis Reuter 123, 124, 126, 127

Coranus subapterus (De Geer) 215

Coreus marginatus (Linnaeus) 215

Coriomeris denticulatus (Scopoli) 215

- scabricornis (Panzer) 215

Corixa dentipes (Thomson) 215

- punctata (Illiger) 215

Corizus hyoscyami (Linnaeus) 215

Cremnocephalus albolineatus Reuter 215

Criocoris crassicornis (Hahn) 210, 215

- quadrimaculatus (Fallén) 215

Cyllecoris histrionicus (Linnaeus) 215

Cymatia bonsdorffi (C. Sahlberg) 215

- coleoptrata (Fabricius) 215

Cymus aurescens Distant 215
Cymus claviculus (Fallén) 215

- glandicolor Hahn 215

- melanocephalus Fieber 215

Cyrtorhinus caricis (Fallén) 215

Deraeocoris punctulatus (Fallén) 215

- scutellaris (Fabricius) 215

Derephysia foliacea (Fallén) 215

Dichrooscytus rufipennis (Fallén) 215

Dictyla convergens (Herrich-Schäffer) 215

- echii (Schrank) 215

Dicyphus constrictus (Boheman) 210, 215

- globulifer (Fallén) 210, 215

Dolichonabis limbatus (Dahlbom) 215

- lineatus (Dahlbom) 215

Dolycoris baccarum (Linnaeus) 215

Drymus brunneus (R. F. Sahlberg) 215

- pilicornis (Mulsant \& Rey) 215

- ryei Douglas \& Scott 215

- sylvaticus (Fabricius) 215

Dryophilocoris flavoquadrimaculatus (De Geer) 215

Elasmostethus brevis Lindberg 215

- interstinctus (Linnaeus) 215

Elasmucha ferrugata (Fabricius) 215

- fieberi (Jakovlev) 215

- grisea (Linnaeus) 215

Elatophilus nigrellus 210

- nigricornis (Zetterstedt) 210, 215

- stigmatellus (Zetterstedt) 215

Empicoris culiciformis (De Geer) 216

- vagabundus (Linnaeus) 216

Eremocoris abietis (Linnaeus) 216

- plebejus (Fallén) 216

Eurydema dominulus (Scopoli) 216

- oleracea (Linnaeus) 216

Eurygaster maura (Linnaeus) 216

- testudinaria (Geoffroy) 216

Euryopicoris fennicus E. Wagner 216

- nitidus (Meyer-Dür) 216

Fieberocapsus flaveolus (Reuter) 216

Galeatus spinifrons (Fallén) 216

Gastrodes abietum Bergroth 216

- grossipes (De Geer) 216

Geocoris ater (Fabricius) 216

- dispar (Waga) 216

- lapponicus Zetterstedt 216

Gerris argentatus Schummel 216

- lacustris (Linnaeus) 216

- lateralis Schummel 216

- najas (De Geer) 216

- odontogaster (Zetterstedt) 216

- paludum Fabricius 216

- sphagnetorum Gaunitz 216

- thoracicus Schummel 216

Glaenocorisa propinqua (Fieber) 210, 216

Globiceps flavomaculatus (Fabricius) 216

- salicicola Reuter 216

Gonianotus marginepunctatus (Wolff) 216

Hallodapus rufescens (Burmeister) 216

Halosalda lateralis (Fallén) 216

Halticus aptcrus (Linnaeus) 216

- major E. Wagner 216

Hebrus pusillus (Fallén) 216

- ruficeps Thomson 216

Hesperocorixa castanea (Thomson) 216

- linnaei (Fieber) 216

- sahlbergi (Fieber) 216

Heterogaster urticae (Fabricius) 216

Holcostethus vernalis (Wolff) 216

Hoplomachus thunbergi (Fallén) 216

Hydrometra gracilenta Horvath 216 
Hydrometa stagnorum (Linnaeus) 216

Ischnocoris angustulus (Boheman) 216 Ischnodemus sabuleti (Fallén) 216

Jalla dumosa (Linnaeus) 216

Kalama tricornis (Schrank) 216

Kleidocerys resedae (Panzer) 216

Labops sahlbergi (Fallén) 216

Lamproplax picea (Flor) 216

Legnotus picipes (Fallén) 216

Leptopterna dolabrata (Linnaeus) 216

- ferrugata (Fallén) 216

Ligyrocoris sylvestris (Linnaeus) 216

Limnoporus rufoscutellatus (Latreille) 216

Liocoris tripustulatus (Fabricius) 216

Lopus decolor (Fallén) 216

Loricula elegantula (Bärensprung) 216

- pselaphiformis Curtis 216

Lyctocoris campestris (Fabricius) 216

- nidicola E. Wagner 216

Lygaeus equestris (Linnaeus) 216

Lygocoris contaminatus (Fallén) 216

- limbatus (Fallén) 216

- lucorum (Meyer-Dür) 216

- pabulinus (Linnaeus) 216

- rhamnicola (Reuter) 216

- spinolae (Meyer-Dür) 216

- viridis (Fallén) 216

Lygus gemellatus (Herrich-Schäffer) 216

- pratensis (Linnaeus) 217

- punctatus (Zetterstedt) 217

- rugulipennis Poppius 217

- wagneri Remane 217

Macrodema micropterum (Curtis) 217

Macrolophus nubilus (Herrich-Schäffer) 217

Macroplax preyssleri (Fieber) 217

Macrosaldula scotica (Curtis) 217

Macrosandalus Stål 139

Macrotylus cruciatus (R.F. Sahlberg) 217

Malacocoris chlorizans (Panzer) 217

Malthacosoma Reuter 46

Mecomma ambulans (Fallén) 217

- dispar (Boheman) 217

Megacoelum infusum (Herrich-Schäffer) 217

Megaloceraea recticornis (Geoffroy) 217

Megalocoleus Reuter 45

- molliculus (Fallén) 217

- pilosus (Schrank) 217

Megalonotus antennatus (Schilling) 217

- chiragra (Fabricius) 217

Mesovelia furcata Mulsant \& Rey 217

Metatropis rufescens (Herrich-Schäffer) 217

Micracanthia fennica (Reuter) 217

- marginalis (Fallén) 217

Micronecta griseola Horvath 217

- minutissima (Linnaeus) 217

- poweri (Douglas \& Scott) 217

Microvelia reticulata (Burmeister) 217

- umbricola Wroblewski 217

Miris striatus (Linnaeus) 217

Monalocoris filicis (Linnaeus) 217

Monosynamma bohemani (Fallén) 210, 217

Myrmecophyes alboornatus (Stål) 217

Myrmecoris gracilis (R.F. Sahlberg) 217

Myrmedobia coleoptrata (Fallén) 217

- distinguenda Reuter 217

- exilis (Fallén) 217

Myrmus miriformis (Fallén) 217

Nabis brevis Scholtz 217

- ericetorum Scholtz 217

- ferus (Linnaeus) 217
Nabis flavomarginatus Scholtz 217

- inscriptus (Kirby) 217

- pseudoferus Remane 217

- punctatus (Costa) 217

- rugosus (Linnaeus) 217

Neides tipularius (Linnaeus) 217

Nemocoris fallenii R.F. Sahlberg 217

Neomecomma bilineatum (Fallén) 217

Neottiglossa pusilla (Gmelin) 217

Nepa cinerea Linnaeus 217

Nithecus jacobaecie (Schilling) 217

Notonecta glauca Linnaeus 217

- lutea Müller 217

- reuteri Hungerford 217

Notostira elongata (Geoffroy) 217

- erratica (Linnaeus) 217

Nysius ericae (Schilling) 217

- groenlandicus (Zetterstedt) 217

- helveticus (Herrich-Schäffer) 217

- thymi (Wolff) 217

Ochetostethus opacus (Scholtz) 217

Oeciacus hirundinis (Jenyns) 217

Oncotylus punctipes Reuter 210, 217

Orius agilis (Flor) 217

- horvathi (Reuter) 217

- niger (Wolff) 217

Orthocephalus brevis (Panzer) 217

- coriaceus 210

- saltator (Hahn) 217

- vittipennis (Herrich-Schäffer) 210, 217

Ortholomus punctipennis (Herrich-Schäffer) 217

Orthonotus rufifrons (Fallén) 217

Orthops basalis (Costa) 218

- campestris (Linnaeus) 218

- kalmi (Linnaeus) 218

Orthotylus boreellus (Zetterstedt) 218

- diaphanus (Kirschbaum) 218

- ericetorum (Fallén) 218

- flavinervis (Kirschbaum) 218

- flavosparsus (C. Sahlberg) 218

- fuscescens (Kirschbaum) 218

- leokhares Linnavuori sp. n. 17

- marginalis Reuter 218

- nassatus (Fabricius) 218

- obscurus Reuter 17

- prasinus (Fallén) 218

- psalloides Wagner 17

- tenellus (Fallén) 218

- virens (Fallén) 218

- viridinervis (Kirschbaum) 218

Oxycarenus modestus (Fallén) 218

Pachybrachius fracticollis (Schilling) 218

- luridus (Hahn) 218

Pachycoleus pusillimus J. Sahlberg 218

- waltli Fieber 218

Pachytomella parallela (Meyer-Dür) 218

Palomena prasina (Linnaeus) 218

Pantilius tunicatus (Fabricius) 218

Paracorixa concinna (Fieber) 218

Peirates sulcicollis Serville 139

Pentatoma rufipes (Linnaeus) 218

Peritrechus angusticollis (R.F. Sahlberg) 218

- convivus (Stål) 218

- geniculatus (Hahn) 218

- nubilus (Fallén) 218

Philomyrmex insignis R.F. Sahlberg 218

Phimodera humeralis (Dalman) 218

- lapponica (Zetterstedt) 218

Phoenicocoris modestus (Meyer-Dür) 218

- obscurellus (Fallén) 218 
Phylus coryli (Linnaeus) 218

- melanocephalus (Linnaeus) 218

Phymata crassipes (Fabricius) 218

Physatocheila costata (Fabricius) 218

Phytocoris dimidiatus Kirschbaum 218

- insignis Reuter 218

- intricatus Flor 218

- longipennis Flor 218

- pini Kirschbaum 218

- populi (Linnaeus) 218

- tiliae (Fabricius) 218

- ulmi (Linnaeus) 218

Picromerus bidens (Linnaeus) 218

Piesma capitatum (Wolff) 218

- maculatum (Laporte de Castelnau) 218

- quadratum (Fieber) 218

Pilophorus cinnamopterus (Kirschbaum) 218

- clavatus (Linnaeus) 218

- confusus (Kirschbaum) 218

- perplexus (Douglas \& Scott) 218

Pinalitus cervinus (Herrich-Schäffer) 218

- rubricatus (Fallén) 218

Pionosomus varius (Wolff) 218

Pithanus hrabei Stehlik 210, 218

- maerkeli (Herrich-Schäffer) 218

Pityopsallus lapponicus (Reuter) 211, 218

- luridus (Reuter) 218

Placochilus seladonicus (Fallén) 218

Plagiognathus albipennis (Fallén) 218

- arbustorum (Fabricius) 218

- chrysanthemi (Wolff) 218

- vitellinus (Scholtz) 218

Plesiocoris rugicollis (Fallén) 218

Plesiodema pinetellum (Zetterstedt) 218

Plinthisus brevipennis (Latreille) 218

- pusillus (Scholtz) 218

Polymerus cognatus (Fieber) 219

- lammesi Rinne 219

- microphthalmus (E. Wagner) 219

- nigritus (Fallén) 219

- palustris (Reuter) 219

- tepastus Rinne 219

- unifasciatus (Fabricius) 219

- vulneratus (Wolff) 219

Psallus aethiops (Zetterstedt) 219

- alnicola 211

- ambiguus (Fallén) 219

- betuleti (Fallén) 219

- falleni Reuter 219

- graminicola (Zetterstedt) 219

- haematodes (Gmelin) 219

- kolenatii (Flor) 219

- lepidus (Fieber) 219

- mollis (Mulsant \& Rey) 219

- perrisi (Mulsant \& Rey) 219

- quercus (Kirschbaum) 219

- scholtzi Fieber 211,219

- variabilis (Fallén) 219

- varians (Herrich-Schäffer) 219

- wagneri Ossiannilsson 219

Pterotmetus staphyliniformis (Schilling) 219

Pygolampis bidentata (Goeze) 219

Ranatra linearis (Linnaeus) 219

Rasahus Amyot \& Serville 131, 139

- aeneus (Walker) 131, 132, 135́, 138, 140

- albomaculatus Mayr 131, 132, 136, 138, 140

- amapaensis Coscarón 131, 132, 136, 138, 144

- angulatus Coscarón 131, 132, 136, 138, 144

- arcitenens Stål 131, 132, 136-138, 144

- arcuiger (Stål) 131, 132, 136, 138, 144
Rasahus argentinensis Coscarón 131, 132, 136, 138, 144

- atratus Coscarón 131, 132, 136, 138, 140

- bifurcatus Champion 131, 132, 136, 138, 140

- biguttatus (Say) 131, 132, 136, 138, 144

- brasiliensis Coscarón 131, 132, 136, 138, 140

- castaneus Coscarón 131, 132, 136, 138, 140

- costarricensis Coscarón 131, 132, 136, 138

- flavovittatus Stål 131, 132, 136, 138, 140

- grandis Fallou 131, 132, 136, 138, 142, 144

- guttipennis (Stål) 131, 132, 136, 138, 140

- hamatus (Fabricius) 131, 132, 136, 138, 142, 144

- limai Pinto 131, 132, 136, 138, 144

- maculipennis (Lepelletier \& Serville) 131, 132, 136, 138 , 140

- paraguayensis Coscarón 131, 132, 136, 138, 140, 141

- peruensis Coscarón 131, 132, 136, 138, 140, 141

- rufiventris (Walker) 131, 132, 136, 138, 144

- scutellaris (Fabricius) 131, 132, 136, 138, 140, 141

- sulcicollis (Serville) 131, 132, 136, 138, 140

- surinamensis Coscarón 131, 132, 136, 138, 140

- thoracicus Stål 131, 132, 136, 138, 144

- vittatus Coscarón 131, 132, 136-138, 143, 144

Rhacognathus punctatus (Linnaeus) 219

Rhinocoris annulatus (Linnaeus) 219

Rhopalus conspersus (Fieber) 219

- parumpunctatus (Schilling) 219

- subrufus (Gmelin) 219

Rhyparochromus phoeniceus (Rossi) 219

- pini (Linnaeus) 219

- (Raglius) maroccanus Wagner 19

- (R.) simplex (Jakovlev) 19

- (R. ) tisifone Linnavuori sp. n. 17-19

- (R. ) vulgaris (Schilling) 19

Salda littoralis (Linnaeus) 219

- morio Zetterstedt 219

- muelleri (Gmelin) 219

- sahlbergi Reuter 219

Saldula arenicola (Scholtz) 219

- fucicola (J. Sahlberg) 219

- opacula (Zetterstedt) 219

- orthochila (Fieber) 219

- pallipes (Fabricius) 219

- palustris (Douglas) 219

- pilosella (Thomson) 219

- saltatoria (Linnaeus) 219

Salicarus roseri (Herrich-Schäffer) 219

Sciocoris cursitans (Fabricius) 219

- microphthalmus Flor 219

- umbrinus (Wolff) 219

Scoloposcelis obscurella (Zetterstedt) 219

- pulchella (Zetterstedt) 219

Scolopostethus affinis (Schilling) 219

- decoratus (Hahn) 219

- pictus (Schilling) 219

- pilosus Reuter 219

- thomsoni Reuter 219

Sehirus luctuosus Mulsant \& Rey 219

Sigara distincta (Fieber) 219

- falleni (Fieber) 219

- fallenoidea (Hungerford) 219

- fossarum (Leach) 219

- hellensii (C. Sahlberg) 219

- lateralis (Leach) 219

- limitata (Fieber) 219

- longipalis (J. Sahlberg) 219

- nigrolineata (Fieber) 219

- semistriata (Fieber) 219

- striata (Linnaeus) 219

Solenoxyphus Reuter 45, 46

Spathocera dalmani (Schilling) 219 
Sphodrocoris Stål 139

Sphragisticus nebulosus (Fallén) 219

Stagonomus pusillus (Herrich-Schäffer) 220

Stalia boops (Schiödte) 211, 220

Stenodema calcarata (Fallén) 220

- holsata (Fabricius) 220

- laevigata (Linnaeus) 220

- trispinosa Reuter 220

- virens (Linnaeus) 220

Stenotus binotatus (Fabricius) 220

Stephanitis oberti (Kolenati) 220

Stethoconus cyrtopeltis (Flor) 220

Sthenarus rottermundi (Scholtz) 220

Stictopleurus abutilon (Rossi) 220

- crassicornis (Linnaeus) 220

- punctatonervosus (Goeze) 220

Strongylocoris leucocephalus (Linnaeus) 220

- niger (Herrich-Schäffer) 220

- steganoides J. Sahlberg 220

Stygnocoris fuligineus (Geoffroy) 220

- pygmaeus (R.F. Sahlberg) 220

- rusticus (Fallén) 220

- sabulosus (Schilling) 220

Systellonotus triguttatus (Linnaeus) 220

Taeniophorus Linnavuori 45, 46, 63

- grandoculus Linnavuori 45

- hyalinus Linnavuori 45, 57, 60, 61, 63

Taphropeltus hamulatus (Thomson) 220

Teloleuca bifasciata (Thomson) 220

- pellucens (Fabricius) 220

Temnostethus gracilis (Horvath) 220

- pusillus (Herrich-Schäffer) 220

Teratocoris antennatus (Boheman) 220

- caricis Kirkaldy 220

- paludum J. Sahlberg 220

- saundersi Douglas \& Scott 211, 220

- viridis Douglas \& Scott 220

Tetraphleps aterrimus (J. Sahlberg) 220

- bicuspis (Herrich-Schäffer) 220

Thyreocoris scarabaeoides (Linnaeus) 220

Tingis cardui (Linnaeus) 220

Trapezonotus anorus (Flor) 220

- arenarius (Linnaeus) 220

- desertus Seidenstücker 220

Trigonotylus coelestialium (Kirkaldy) 220

- fuscitarsis Lammes 220

- ruficornis (Geoffroy) 220

Tritomegas bicolor (Linnaeus) 220

Troilus luridus (Fabricius) 220

Tropistethus holosericeus (Scholtz) 220

Tytthus geminus (Flor) 220

- pygmaeus (Zetterstedt) 220

Ulmicola spinipes (Fallén) 220

Velia saulii Tamanini 220

Xylocoris cursitans (Fallén) 211, 220

- formicetorum (Boheman) 220

- galactinus (Fallén) 220

- lativentris (J. Sahlberg) 220

- parvulus (Reuter) 220

Zicrona caerulea (Linnaeus) 220

\section{Homoptera}

Acyrthosiphon (Liporrhinus) chelidonii (Kaltenbach) 86, 98

- (Metopolophium) graminearum Mordvilko 94

- aurlandicum Heikinheimo 65, 85

- brachysiphon Hille Ris Lambers 65, 84, 98

- knechteli (Börner) 85, 98
Anuraphis farfarae (Koch) 76

Aphis avenae Fabricius 94

- dirhoda Walker 94

- granaria Kirby 94

Aulacorthum flavum Müller 81, 82, 98

- speyeri Börner $82,83,98$

- vaccinii Hille Ris Lambers 83, 98

Capitophorus carduinus (Walker) 76, 98

- elaeagni (Del Guercio) 76

Cryptaphis poae (Hardy) 88, 89, 98

- setiger Hille Ris Lambers 88

Diuraphis (Holcaphis) calamagrostis (Ossiannilsson) 68, 69,98

- (H.) frequens (Walker) 63, 69

Dysaphis aizenbergi (Shaposhnikov) 65, 66, 98

- hirsutissima Börner 67,98

- newskyi (Böner) 65

- ossiannilssoni Stroyan 65

Ericaphis Börner 73

- empetri 73

Fimbriaphis fimbriata Richards 73

- lagarriguei Börner 73

- latifrons (Börner) 73, 74, 76, 98

Hyadaphis foeniculi Passerini 69, 70

- mellifera Hottes 69, 70

- passerinii (del Guercio) 69, 70, 98

- polonica Szelegiewicz 69, 70, 98

- sphondylii (Koch) 70

Hydaphias mosana Hille Ris Lambers 70, 72, 98

Hyperomyzus (Neonasonovia) sobrinus Müller 80

- (N.) thorsteinni Stroyan 79, 80

- (N.) zirnitsi boerneri Prevost 79, 80, 98

- (N.) zirnitsi Hille Ris Lambers 79

Idiovatus Börner 73

Javesella pellucida (Fabricius) 149

Macrosiphum 90

- (Sitobion) scoticum Stroyan 94

Metadelphax propinqua (Fieber) 145-149

Metopolophium Mordvilko 73, 90

- beiquei Hille Ris Lambers 94

- brevirostre Heikinheimo 94

- dirhodum (Walker) 87

- festucae (Theobald) 87, 88

- graminearum Mordvilkko 94

- tenerum Hille Ris Lambers 87, 88, 98

Myzodium Börner 73

- lagarriguei Remaudiere 73

- modestum (Hottes) 76, 98

Myzus (Nectarosiphon) 74

Nasonovia (Kakimia) saxifragae (Doncaster \& Stroyan) 99101

- (K.) vannesi Stenseth 78, 98

Neodecorosiphon muscicolens Heinze 88

Nilaparvata lugens (Stål) 149

Ovatus van der Groot 73

Pseudacaudella rubida Börner 72, 73, 98

Rhodobium porosum (Sanderson) 90, 92, 98

Rhopalosiphonius (Pseudorhopalosiphonius) calthae Koch $80,81,98$

Schizomyzuz lindneri Börner 73

Sitobion (Metobion) Heikinheimo 65

- (Metobion) graminearum (Mordvilko) 65, 93, 94, 98

- dryopteridis (Holman) 92,98

- scoticum Stroyan 94

Stiroma bicarinata (Herrich-Schäffer) 146

Subacyrthosiphon cryptobium Hille Ris Lambers 90, 91, 98

Uroleucon (Uromelan) Hille Ris Lambers 95

- (U.) riparium (Stroyan) 95, 98

- jaceae 95

- solidaginis 95 


\section{Hymenoptera}

Aclastus caudator Hellén 181, 134

- furcifer Hellén 185

- gracilis (Thomson) 185

- micator (Gravenhorst) 184

- opacinotum Hellén 185

- planicollis Hellén 185

- setosus Hellén 181, 184, 185

Alegina thunebergi Hellén 184

Apsilops cictorius var. tenebrosus Hellén 183

- cinctorius (Gravenhorst) 183

- tenebrosus Hellén 183

Arotrephes glabriculus (Hellén) 184

- nivosus (Hellén) 184

Asyncrita canaliculata Hellén 181

- scutellata Hellén 182

Atractodes nigripennis Hellén 182

Bathythrix maculata (Hellén) 183

Brachycephalus tenerifae Hellén 182

- tenerifae var. thoracicus Hellén 182

Caenocryptus melanarius Hellén

Catalytus fennicus Hellén 185

Charitopes Förster 184

- breviceps Hellén 184

- brevistylus Hellén 184

- curticauda Hellén 184

- macrocerus Hellén 184

Clypeoteles distans (Thomson) 185

Dichrogaster tenerifae (Hellén) 182

Diprion pini (Linnaeus) 175-178

"Ethelurgus" curticauda (Hellén) 184

Formica cinerea Mayr 192

- fusca Linnaeus 192

- pratensis Retzius 192

- pressilabris Nylander 192

Formicoxenus nitidulus (Nylander) 191

Gelis balteata (Thomson) 184

- breviceps (Hellén) 184

- cephalotes Hellén 185

- inflatipes Hellén 185

- lapponica Hellén 185

- lapponicus Hellén 185

- speciosa (Hellén) 182

Gilpinia socia (Klug) 175-178

Habrocryptus canariensis Hellén 182

Harpagoxenus sublaevis (Nylarder) 191

Hedylus crassicornis Ashmead 181, 185

Hemiteles Gravenhorst 181

- rufigaster Horstmann nom. nov. 181, 182

- rufiventris Hellén 181, 182

- similis (Gmelin) 182

- speciosus Hellén 182

Hidryta Förster 182

- atlantica Horstmann sp. n. 181, 185

- pardosae Nishida 185

Idiolispa analis f. nigriventris Habermehl 182

- analis Gravenhorst 182

- morio Hellén 181, 182, 185

- vagabunda Seyrig 182

Isadelphus Förster 184

- parviceps Hellén 184

- pusillus Hellén 184
Ischnurgops pellucidator maculatus Hellén 183

Ischnus canariensis (Hellén) 182

Ischnus porrectorius var. melanarius Hellén 183

Iselix elfvingi Hellén 181, 183

Lasius mixtus (Nylander) 191

Leptogenys sp. 22

Leptogenys mutabilis (Smith) 22

Leptothorax muscorum (Nylander) 191

Mastrus parviceps (Hellén) 184

Myrmica rugulosa Nylander 191

- sabuleti Meinert 191

- scabrinodis Nylander 191

- schencki Emery 191

- sulcinodis Nylander 191

Neodiprion sertifer (Geoffroy) 175-178

Orthizema Förster 185

- macrocerum (Hellén) 184

- opacinotum (Hellén) 185

- pullator (Gravenhorst) 185

Peristenus Förster 189

- digoneutis Loan 189-190

- pallipes (Curtis) 189-190

- rubricollis Thomson 189-190

- stenodemae Loan 189-190

- stygicus Loan 189-190

Pheidologeton diversus 22

Phygadeuon Gravenhorst 181

- austriacus Gravenhorst 181, 183

- inaris (Hellén) 183

- lateareolatus Hellén 183

- planicollis (Hellén) 185

- ponojensis (Hellén) 183

- striiventris Hellén 183

Phyzelus boreophilus Hellén 183

- glabriculus Hellén 184

- inaris Hellén 183

- nivosus Hellén 184

- ponojensis Hellén 183

Pleurogyrus pumilus (Hellén) 184

Sulcarius thunebergi (Hellén) 184

Theroscopus Förster 185

- boreophilus (Hellén) 183

Trychosis Förster 182

Uchidella longicauda Hellén 184

- pumila Hellén 184

Xiphulcus floricolator (Gravenhorst) 184

\section{Lepidoptera}

Bucculatrix latviaella Šulcs, sp. n. 41-43

- maritima Stainton 41-43

Noctua pronuba (Linnaeus) 155-157, 159-161

\section{Siphonaptera}

Amphipsylla rossica Wagner 180

Ceratophyllus farreri Rothschild 180

- hirundis Curtis 180

Ctenophthalmus assimilis Taschenberg 180

- bisoctodentatus Kolenati 180 